\section{Insektengiftallergie frühzeitig behandeln!}

\author{
„Insektengiftallergien bei Kindern wachsen sich mit den Jahren \\ schon aus." Diese Annahme ist weit verbreitet - und leider sehr oft \\ falsch, wie eine retrospektive Erhebung aus den USA zeigt.
}

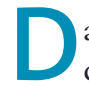
avid Golden und sein Team von der Johns Hopkins University, Baltimore, schrieben über 1.000 Patienten an, bei denen im Kindesalter zwischen 1978 und 1985 eine Insektengiftallergie diagnostiziert worden war. Von den 512 Patienten, die antworteten, hatte ein knappes Drittel (163) eine spezifische Immuntherapie über einen Zeitraum von 3,5 Jahren erhalten.

In der nachfolgenden Zeit waren $43 \%$ der insgesamt 512 Patienten wieder von Insekten gestochen worden. Bei denen, die in der Kindheit hyposensibilisiert worden waren, traten nur zu 3\% systemische Reaktionen auf. Hatten die Patienten dagegen keine Immuntherapie erhalten, so lag die Häufigkeit systemischer allergischer Reaktionen bei $17 \%$. Das Risiko nahm in dieser Grup-

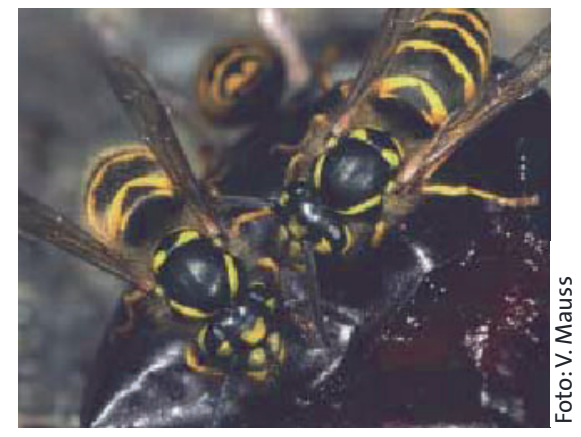

Achtung Anaphylaxiegefahr! Bei jedem 100. Kind führt ein Wespen- oder Bienenstich zu systemischen Reaktionen.

pe zwar im Lauf der Zeit etwas ab, die Häufigkeit betrug aber nach 20 Jahren immer noch $13 \%$.

Der Effekt der Immuntherapie zeigte sich besonders deutlich in der
Untergruppe der Patienten, die als Kinder mäßige bis schwere anaphylaktische Reaktionen gezeigt hatten: Wurden sie im Erwachsenenalter gestochen, kam es ohne Immuntherapie bei $32 \%$ zu systemischen Reaktionen, nach Immuntherapie war dies nur bei 5\% der Fall.

Von den Patienten, die schon als Kinder nur leichte, auf die Haut beschränkte systemische Symptome aufgewiesen hatten, erlitt nach Hyposensibilisierung keiner als Erwachsener eine anaphylaktische Reaktion, ohne Hyposensibilisierung jedoch $13 \%$.

Fazit: Eine Hyposensibilisierung gegen Insektengift im Kindesalter reduziert das Risiko für spätere anaphylaktische Reaktionen - dieser Effekt hält für den beachtlichen Zeitraum von 10-20 Jahren an. Besonders profitieren Kinder mit initial schweren Reaktionen. jfg

Golden DB et al. Outcomes of allergy to insect stings in children, with and without venom immunotherapy. N Engl J Med 2004; 351: 668-74

\title{
Bienengiftextrakte: wässrig oder Depot?
}

Die Hyposensibilisierung mit Insektengift ist die Therapie der Wahl für Patienten mit anaphylaktischen Reaktionen nach Bienenstichen. Eine prospektive Studie verglich jetzt erstmals Wirksamkeit und Verträglichkeit verschiedener Giftpräparationen und Therapieregime.

M ünchner Allergologen wiesen 65 Patienten mit einer Bienengiftallergie einem von drei Behandlungsschemata zu: Patienten der Gruppen A und B wurde im Rahmen eines Rush-Protokolls in der Steigerungsphase eine wässrige Bienengiftzubereitung subkutan appliziert, Patienten der Gruppe $\mathrm{C}$ in einem konventionellen, „langsamen“ Steigerungsverfahren eine Aluminiumhydroxid-Depotformulierung. In der Erhaltungsphase erhielten alle Patienten jeweils $100 \mu \mathrm{g}$ Gift pro Injektion, Gruppe $\mathrm{A}$ in Form der wässrigen Formulierung alle 4 Wochen, die Gruppen B und C als Depotformulierung, wobei das Injektionsintervall bei Gruppe B 8 Wochen und bei Gruppe C 4 Wochen betrug.
Gesteigerte Lokalreaktionen (über $20 \mathrm{~cm}$ Durchmesser) traten insgesamt häufiger unter dem wässrigen Insektengift als unter dem Depot-Insektengift auf - und zwar sowohl während der Steigerungsphase $(7,6 \%$ vs. $3,9 \%, p=0,059)$ als auch während der Erhaltungsphase ( $49,4 \%$ vs. $12,1 \%, \mathrm{p}<0,001)$. Systemische Nebenwirkungen während der Steigerungsphase schienen häufiger unter dem Rush-Protokoll aufzutreten (31,0\% vs. $13,0 \%$, nicht signifikant).

Bei Reexposition (49-mal durch Stichprovokation, siebenmal durch Feldstiche) traten bei drei von 20 Patienten der Gruppe A (15,0\%), bei zwei von 18 Patienten der Gruppe B (11,1\%) und bei drei von 18 Patienten der Gruppe C
(16,7\%) erneut systemische anaphylaktische Reaktionen auf (Unterschiede nicht signifikant).

Ursache der häufigeren gesteigerten Lokalreaktionen bei Verwendung eines wässrigen Extraktes könnte die langsamere Allergenfreisetzung aus der Depotzubereitung sein. Die erzielte Schutzrate von nur etwa $85 \%$ ist bei einer Bienengift-Hyposensibilisierung nicht ungewöhnlich - bei einigen Patienten reicht die Erhaltungsdosis von $100 \mu \mathrm{g}$ nicht aus, hier muss dann die Behandlung mit höherer Dosis weitergeführt werden.

Fazit: Eine Immuntherapie mit wässrigen Bienengiftzubereitungen führt vermehrt zu gesteigerten Lokalreaktionen. Jedoch ist eine Rush-Hyposensibilisierung für viele Patienten zum raschen Aufbau eines Schutzes unerlässlich. $\quad b k$

Ruëff F et al. Specific immunotherapy in honeybee venom allergy: a comparative study using aqueous and aluminium hydroxide adsorbed preparations. Allergy 2004; 59: 589-95 